\title{
Emotype: Expressing emotions by changing typeface in mobile messenger texting
}

\author{
Saemi Choi ${ }^{1}$ (D) Kiyoharu Aizawa ${ }^{1}$
}

Received: 29 January 2018 / Revised: 1 August 2018 / Accepted: 3 October 2018 /

Published online: 31 October 2018

(C) The Author(s) 2018

\begin{abstract}
Instant messaging is a popular form of text-based communication. However, text-based messaging lacks the ability to communicate nonverbal information such as that conveyed through facial expressions and voice tones, although a multitude of emotions may underlie the text of a conversation between participants. In this paper, we propose an approach that uses typefaces to communicate emotions. We investigated which typefaces are useful for delivering emotions and introduced these typefaces into a mobile chat app. We conducted a survey to demonstrate how changes in the typeface of a message affected the meaning of the message conveyed. Our user study provides an understanding of the actual user experience with the application. The results show that the use of multiple typefaces in a message can affect and intensify the valence received by users and the use of multiple typefaces elicited an active response and brought about a livelier mood during texting.
\end{abstract}

Keywords Typeface $\cdot$ Font $\cdot$ Emotion · Computer-mediate-communication ·

Mobile messenger

\section{Introduction}

The sharing of emotions among people can elicit empathy, increase a friendly feeling, and even improve mental health [14]. In face-to-face situations, people can express their emotions or feelings with facial expressions, voice tones, gestures, and so on. The function of emotional expressions is not only to express one's inner state, but also to guide a situation toward an intended mood [15]. For example, if someone asks, "Could you do me a favor?" with a smile, the mood of the situation may be positive, and the receiver of the question would likely be more willing to respond affirmatively. However, if the person makes the

Saemi Choi

merrycsm@hal.t.u-tokyo.ac.jp

Kiyoharu Aizawa

aizawa@hal.t.u-tokyo.ac.jp

1 Faculty of Engineering Building 2, The Univeristy of Tokyo, 7-3-1 Hongo, Bunkyo-ku, Tokyo 113-8656, Japan 
request in an overbearing tone of voice, the mood may be negative, and the receiver may feel coerced to respond. In this way, nonverbal signals can change the receiver's attitude, which can affect his or her reaction to the situation. Despite the importance of nonverbal cues in effective communication, computer-mediated communication tools such as text-based communication (e-mail or text messaging apps) inherently lack nonverbal signals for conveying emotional information [45].

As the text-based communication become popular, alternate ways for expressing nonverbal signals such as emoji have developed. These pictorial representations are contributing to our communication engagement by making the communication more lively. Meanwhile, the effect of typeface has been studied in the design and marketing literature such as emotional response to logos and advertisements [24, 32], preference for use of certain fonts [43], and the relationship between the visual appearance and emotional responses in fonts [3]. However, the role of typeface has been limited to marketing, so that only experts, such as designers, have considered the use of typefaces as a communication medium.

In this paper, we propose Emotype, a mobile messenger application prototype that enables general users to change the typeface of a mobile messenger message to convey certain emotions (Fig. 1). The novelty of this study is to generate a new value of typeface in the context of the mobile messaging, where the role of typeface has not been investigated. Even though there are already various ways of expressing emotions in mobile messengers (emoticon/emoji, voice message, and so on), introducing a new system using typeface will bring a richer communication experience. We expect our prototype enables not only communication experts but also general users to become aware of the emotional function of the typeface so that they actively engage in font communication.

The contributions of this study are outlined below. We:

- build a mobile messenger application prototype that enables users to communicate with typefaces;

- demonstrate the feasibility of typefaces for communicating emotions with a survey study; and

- $\quad$ explore the unique feature of typeface different from other ways for expressing emotion by qualitative user study.

As far as we know, this paper is the first work to explore the effect of typefaces for message texting. We therefore focus on the basic emotion scale (negative, positive and neutral [19]) to observe the sharp contrast of effects between negative and positive fonts. In the following section, we review related studies about typefaces in communication. We then present the Emotype application prototype and introduce the emotion-bearing typefaces adopted in the prototype. Our user study design is then introduced. We investigate the effect of the message content in combination with positive and negative typefaces and present

Fig. 1 User interface of the Emotype prototype. A user can send a text message and change the message typeface to convey a particular feeling

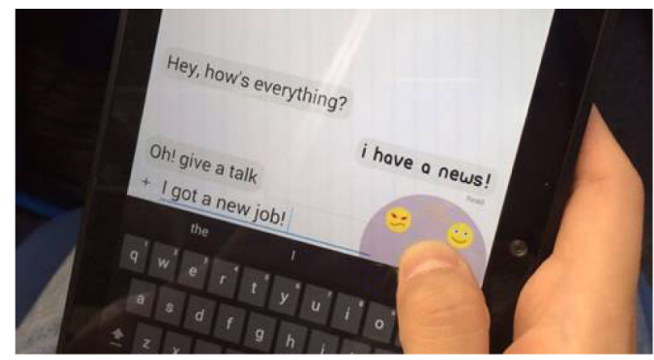


various and unique user behaviors, experiences, and user comments obtained from the user study.

\section{Related work}

\subsection{Nonverbal signals in mobile messengers}

Users of mobile messengers cannot express their emotions through the device using facial expressions, voice tones, or gestures. Nonetheless, alternate ways exist for expressing nonverbal signals in mobile environments. Emoticons, which are a combination of punctuation marks and letters that represent human facial expressions, are one of the most general ways to express nonverbal signals by mobile messenger users (e.g., ":-)", smiley face). In the late 1990s, emoji (e.g., ()) were invented and added to the Unicode system and became increasingly popular with the development of devices supporting graphics, such as smartphones. Emoticons and emoji perform the role of providing emotional signals, thereby improving communication, and conferring a certain mood to the chat [40]. Capitalization of all letters can provoke polarized responses. For example, the use of full capitalization of a positive sentence, such as "HAPPY TO HEAR THAT," conveys extreme joy in contrast to the typical use of upper and lower cases, such as "Happy to hear that." In the same way, capitalization of a negative sentence intensifies the negative feelings [7]. It has also been verified that letter repetition, which extends a syllable, such as "Sweeeet", conveys auditory signals and invokes playful impressions [27]. Moreover, punctuation and quotation marks can be used to convey sarcasm [16], such as “Thank you 'very' much.” It is also known that using more words or being quick to respond can engender a positive impression in the receiver [22]. In addition, the size of the text affects the sense conveyed. For example, the Google Allo messaging app enables users to change the size of the font to denote voice volume [26]. Recently, haptic interface demonstrated that emotions can be transferred by tactile stimuli such as temperature [44].

\subsection{The usage of typeface in multimedia}

The importance of the typeface has been investigated in many fields, including design, marketing, and computer vision. The legibility of a typeface is important for effective communication and has thus been studied for many years [1, 4, 42]. It has been shown that the arrangement of typefaces, e.g., kerning, line spacing, and letter spacing, affect communication. Font size and line spacing are classic issues that affect legibility and comprehensibility of text [41]. In addition to the function of a typeface in effective delivery of content, the impressions created by a typeface have been actively discussed in recent years. Many researchers have suggested that each typeface creates a unique impression, which is also known as the typeface's personality [3, 32, 43]. Shaikh et al. investigated the relationships between personality traits and preference for use of certain fonts [43]. They suggested that sans serif fonts are effective for website text or email, but serif fonts are more effective for business documents. Li and Suen examined the personalities of 24 typefaces and grouped them into four categories - directness, gentleness, cheerfulness, and fearfulness-based on survey data [32]. Amare and Manning explained why specific typeface features elicit certain emotional responses, thereby supporting the findings of previous studies based on empirical user evaluations [3]. These typeface personalities have been studied and utilized in commercial applications, such as advertising and market research. 
As the number of typeface increasing, font retrieval challenge has gained attention. Thanks to the recent advances in computer vision, font identification from real-world text images such as signboard has achieved remarkable performance for both English [46], and Chinese fonts [25]. These systems are helpful when a user has a certain reference image for the typeface to be searched. Search systems that can be applied in another search scenario have also been proposed; exploring fonts using high-level attributes, such as "dramatic" [38] and recommending fonts that match well with users' graphical input such as background image [11]. Recently, by leveraging recent generative adversarial networks (GAN), typeface glyph synthesizing from few samples by separating style from text image has developed [5, 47]. The above studies support the idea that each typeface has its unique style and affects the sentiment of the written text. Differing from those works, we explore emotional effects of typefaces for message texting.

\subsection{Typography communication in mobile messengers}

Several mobile applications exist that enable users to exploit the emotional effects of typefaces. The Font Dresser [36] font editor application, for example, enables users to change the typeface, text color, and so on. However, it requires saving the text of the changed font into an image format to share it. The Font Infinity [33] application employs symbols that

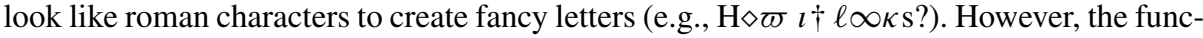
tion of these apps is limited to an aesthetic role. Recently, stickers and illustrations, such as emojis, on Line [13] mobile chat apps provide text with various typefaces in the form of illustrations. The use of OCR font in web-based advising service made users perceive a adviser as a chatbot [8]. Google's Allo is a chat application that allows users to change the size of fonts. Text in a small font size implies a whispering voice, while text in a large font size conveys a raised or shouting voice [26]. Kinetic typography - moving text, emotionally influences the viewer through changes in animation, speed, and dynamics of written text $[28,31]$.

These applications reflect the need for general users to exploit the various effects of typefaces. However, compared with emoji and emoticons, the affect associated with typefaces on the mobile environment has not been studied. Although [28] studied the effects of kinetic typography, there have been few presentations of actual user experiences.

\subsection{Studies on affect and emotion}

Two frequently used psychological emotion models exist: dimensional and categorical. The dimensional model defines emotions in a continuous space in two or three dimensions (valence, arousal or include intensity) [35]. In the categorical model, emotions can be described as discrete categories, e.g., six emotion categories [17] (happiness, sadness, anger, disgust, and fear) and the polarity scale [19] (negative, positive and neutral). These psychological models have been applied not only to recognize emotions from various media, including images, text, and audio [2, 29, 34], but also to collect typeface emotion labels [12].

Many works have studied about various personality traits (or sentiments) of typeface. Some researchers investigated emotions created by typefaces (e.g., happy, angry) based on the general emotional models stated above [39], while others considered impressions [32] (e.g., friendly, reliable). Other studies have examined the function of typefaces for delivering content $[10,42]$ (e.g., readability and memorability). 
In this paper, we investigate whether typeface contributes to change the state of valence and intensifies the emotion a message conveys. As dimensional model theory (valencearousal) explains, intensified emotions are likely to have a high arousal showing $\mathrm{u}$-shaped curve in the valence-arousal dimension [37]. We, therefore, focus on the polarity of typeface - negative, positive, and neutral, instead of using the models which classify emotions sensitively by the level of arousal.

\section{Material}

\subsection{Emotional typefaces}

Choi et al. developed 100-Font dataset [12] in which each typeface was labeled with six emotion categories (happiness, sadness, surprise, fear, anger, and disgust) by 40 crowdsourcing workers. As we discussed in the related work, this work focuses on the polarity of typeface. Therefore, given the dataset with six emotion categories, we selected two representative emotions for the polarity scale - happiness and anger which are both high arousal emotion. By doing that, we expect to be able to observe the sharp contrast between positive typeface and negative typeface which illustrates effects of the typefaces.

Because the dataset only provided the overall labeling tendency which shows that some typefaces have a high agreement in the labeling result among workers, we investigate to find the fonts which have the most emotional influence in each emotion category. Figure 2 shows the procedure how we found the most emotional font for each emotion category. Because some of the fonts have a low agreement in the labeling results, we firstly filtered out typefaces that have a low emotional consensus (Fig. 2a). Then, in order to select the most emotional font for each emotion among the filtered fonts, a crowdsourcing study was designed (Fig. 2b). We recruited 200 workers from Yahoo crowd sourcing service, and they conducted the two-alternative forced choice (2AFC) task (Fig. 3). Based on the collected workers' assessments, the emotional influence scores were estimated using a pairwise ranking algorithm [9]. Here, we chose anger font among the negative fonts and regarded it as

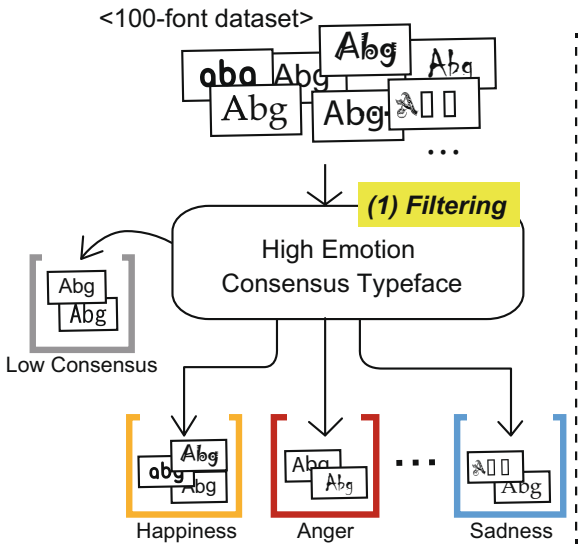

(a) Filtering

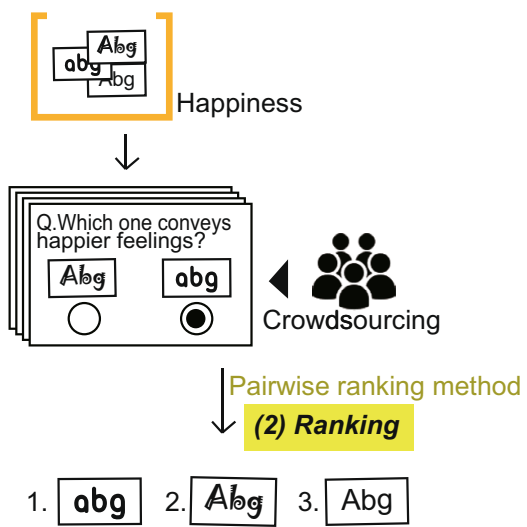

(b) Ranking

Fig. 2 Procedure to select high-emotion typefaces 
Fig. 3 Example of a two-alternative forced choice (2AFC) question

Q. Which one conveys happier feelings?

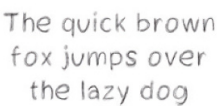

$\mathrm{A}$

\author{
the quick brown \\ fox jumps over \\ the lazy dog
}

(.) $B$

a negative font for the following user studies. In the same way, we regarded the happiness font as a positive font. And the font Arial is selected as a neutral font.

Figure 4 shows the representative typefaces that have the highest emotional influence score for two categories via pairwise ranking study. The positive (happiness) font has rounded caps and a well-balanced appearance. The negative (anger) font has heavily weighted characters on a wild painted background.

\subsection{Messenger application prototype}

We developed a messenger application that enables users to easily change the typeface of a message to reflect their emotion. Figure 5 shows the illustration of the Emotype prototype interface. Emotype was developed based on the Atlas [30] open-source customizable messaging app.

The Emotype mobile application enables users to select a typeface when they want to send a typed message (Fig. 5). When a user applies a long touch on the SEND button, the emotion selection window appears. The user can then move his or her finger to the intended emotion. With these gestures, the typeface of the typed message changes from the neutral to the emotional typeface that the user intended. If the user simply tap the button SEND without the swiping gesture, the message is sent in the Neutral typeface.

\section{Quantitative study: Feasibility of using typefaces for emotion communication}

To demonstrate the feasibility of using typefaces for emotion communication, we tested two null hypotheses:

- $H 0_{1}$ : the typeface used in a message cannot affect the valence (positive or negative) received by users;

- $\mathrm{HO}_{2}$ : the typeface used in an emotive sentence cannot intensify the valence (positive or negative) received by users.

\begin{tabular}{|c|c|c|}
\hline Positive & Neutral & Negative \\
\hline handgloves & Handgloves & 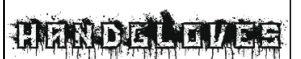 \\
\hline
\end{tabular}

Fig. 4 Representative typefaces for each emotion category 
Fig. 5 Interface for changing the typeface

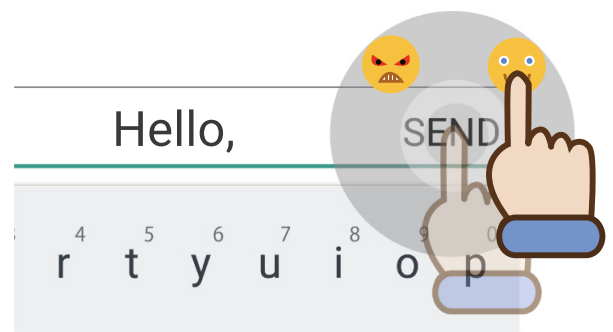

We tested the hypotheses using a questionnaire. We created an online survey that was compatible with both mobile devices and desktop computers. We invited 55 participants who had no background information about the study, and 36 participants (20 males, 16 females) between the ages of 17 and 46 years old (mean $=28.42, \mathrm{SD}=5.07$ ) completed the questionnaire fully.

\subsection{Task}

We provided various conversations to participants (Table 1). Figure $6 \mathrm{a}$ and $\mathrm{b}$ show examples of the tasks for testing $\mathrm{HO}_{1}$ and $\mathrm{HO}_{2}$, respectively. The lines in bold for each conversation indicate the target messages that have variations in the typeface used. Participants were requested to imagine the speaker's facial expression and voice tone during the target message.

\subsubsection{Task 1: Questions for $\mathrm{HO}_{1}$}

We demonstrated that the valence on a message is changed by the typeface used. To do that, we designed the target message to be ambivalent (refer to rows in $H 0_{1}$ of Table 1). Participants were requested to guess the speaker's facial expressions and voice tone during the target message and to pick the option that gave the closest match (-1: negative, 0 : neutral, 1: positive). We prepared three conversations, and each conversation had three variations in the typeface used (negative, neutral, and positive). In total, participants responded to nine questions that were given in random order.

\subsubsection{Task 2: Questions for $\mathrm{HO}_{2}$}

We investigated whether the perceived emotional effect was strengthened by the positive or negative typeface. For that reason, we designed the target message to be negative or positive (refer to rows in $\mathrm{HO}_{2}$ of Table 1). We asked participants to guess how much the speaker expresses negative or positive feelings in the last message and they were requested to respond using a five-point Likert scale (1: not much, 2: a little, 3: somewhat, 4: much, 5: a great deal). We prepared two conversations for each emotion (Positive 1 and 2 for positive, Negative 1 and 2 for negative) and each conversation had two variations in using typeface (neutral or emotional). Consequently, there were eight questions and they were given in random order.

\subsection{Results}

Table 2 shows the mean and standard deviation of the valence ratings by typeface to test $H 0_{1}$. For Neutral 1 and 2, the target message with positive typeface brought about higher 
Table 1 The conversations provided for testing the two hypotheses
The lines in bold for each conversation indicate the target messages that have variations in the typeface used. For $H 0_{1}$, we designed the content of the target message to be neutral with three typeface variations (neutral, positive, and negative). For $\mathrm{HO}_{2}$, the target message was obviously negative or positive, and each has two typeface variations (neutral and negative vs. neutral and positive)

\begin{tabular}{|c|c|c|}
\hline & Task & Conversation \\
\hline \multirow[t]{3}{*}{$H 0_{1}$} & Neutral 1 & $\begin{array}{l}\text { A: I visited an art exhibition } \\
\text { B: Anything interesting? } \\
\text { A: There was a painting of a jar that } \\
\text { was full of pencils. But the artist said } \\
\text { the jar was both full and empty } \\
\text { B: but it was full of pencils! } \\
\text { how could he say it was empty? } \\
\text { A: Artists see things differently }\end{array}$ \\
\hline & Neutral 2 & $\begin{array}{l}\text { A: Today is Friday the } 13 \text { th } \\
\text { B: That's a bad day } \\
\text { A: It's supposed to be unlucky } \\
\text { B: You're supposed to stay home all day } \\
\text { A: That's what I do }\end{array}$ \\
\hline & Neutral 3 & $\begin{array}{l}\text { B: Where are you gonna go? } \\
\text { A: I have to walk the dog } \\
\text { B: What kind of dog do you have? } \\
\text { A: poodle } \\
\text { B: Oh, they bark a lot } \\
\text { A: They sure do }\end{array}$ \\
\hline \multirow[t]{4}{*}{$\mathrm{HO}_{2}$} & Negative 1 & $\begin{array}{l}\text { A: My laptop is so slow } \\
\text { B: Take it to a computer shop } \\
\text { A: no money } \\
\text { B: I see. How about buying a new one } \\
\text { A: I said I have no money }\end{array}$ \\
\hline & Negative 2 & $\begin{array}{l}\text { A: Did you hear about the baseball player? } \\
\text { B: The home run hitter on drugs? } \\
\text { A: Yeah. I'm a big fan of that team... } \\
\text { B: It caused a } \$ 7 \text { million loss to the team } \\
\text { A: I want to beat him up }\end{array}$ \\
\hline & Positive 1 & $\begin{array}{l}\text { B: I am eating a simple salad } \\
\text { A: What do you put in it? } \\
\text { B: Just lettuce, tomato, and celery } \\
\text { A: That's it? } \\
\text { B: Then, I add French dressing } \\
\text { A: That sounds good }\end{array}$ \\
\hline & Positive 2 & $\begin{array}{l}\text { B: Gravity is very important } \\
\text { A: What is gravity? } \\
\text { B: the force that pulls everything down } \\
\text { A: I don't understand } \\
\text { B: You would float into the sky like a balloon } \\
\text { A: That would be fun }\end{array}$ \\
\hline
\end{tabular}




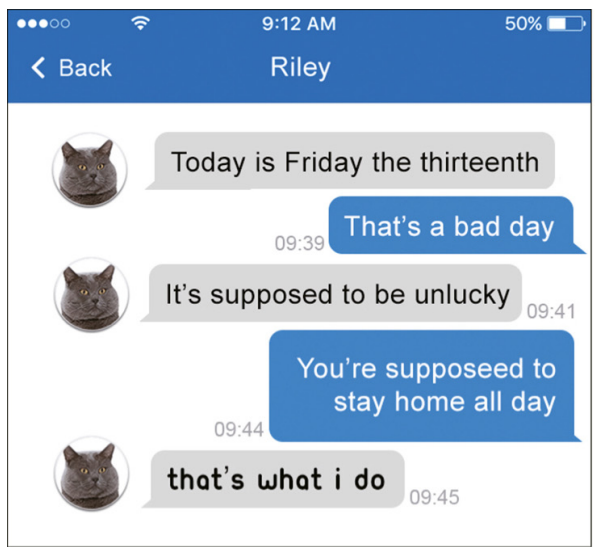

(a) $H 0_{1}$ : Testing whether typefaces affect the valence of a message (Task Neutral 2 in Table 1).
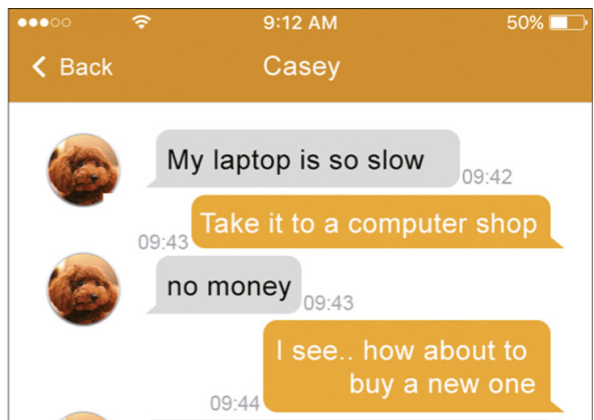

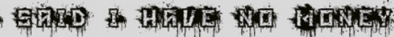

Fig. 6 Examples of conversations for testing each hypothesis

scores than others (higher scores indicate more positive). In case of the message with negative typeface, participants rated lower scores than others (lower scores indicate more negative). For Neutral 3, we could not discover any mean difference between neutral and positive typefaces. However, the target message with negative typeface brought about much lower ratings than those with the neutral typeface. To assess the significance of differences between means for each typeface, one-way ANOVA was conducted (for conversations 1 and 2 at the .01 level). We found a significant effect of typeface on the perceived valence (Neutral 1: $\mathrm{F}(2,105)=20.25 p<.001$, Neutral 2: $\mathrm{F}(2,105)=11.36 p<.001)$.

Table 3 shows the mean and standard deviation of the valence ratings depending on the use of positive or negative typefaces. We discovered that the participants reported higher valence ratings with positive or negative typefaces than with the neutral typeface. To observe each participant's rating with and without typeface, a paired t-test was conducted (at the .05 level). For all the tasks, participants had significantly stronger valence effect with the positive or negative typeface than without it (Negative 1: $p<.001$, Negative $2: p<.001$, Positive 1: $p=.021$, Positive $2: p=.044$ ).

In this section, we conducted a survey study and observed significant differences depending on whether or not the positive or negative typeface was used. Although the contribution of font varies depending on the conversations, we confirmed that the use of fonts changed the meaning of the message. From the results, we can reject the two null hypotheses $H 0_{1}$

Table 2 The values in each cell indicate MEAN (SD)

\begin{tabular}{|c|c|c|c|}
\hline Task & Neutral & Positive & Negative \\
\hline Neutral $1^{* *}$ & $0.08(.43)$ & $0.36(.58)$ & $-0.50(.69)$ \\
\hline Neutral $2^{* *}$ & $-0.06(.47)$ & $0.17(.65)$ & $-0.53(.73)$ \\
\hline Neutral 3 & $0.03(.44)$ & $0.03(.55)$ & $-0.69(.57)$ \\
\hline
\end{tabular}

The target messages with positive or negative typefaces achieved higher positive or negative ratings. Here, a higher score means more positive, a lower score means more negative. The values with an ${ }^{* *}$ mark indicate a statistically significant difference at the .01 level 
Table 3 The results of Task 2

\begin{tabular}{lllll}
\hline & Neutral & Positive & Negative & p-value \\
\hline Negative 1* & $3.53(.90)$ & - & $4.42(.79)$ & $<.001$ \\
Negative 2* & $3.61(.76)$ & - & $4.36(.79)$ & $<.001$ \\
Positive $1^{*}$ & $2.83(0.83)$ & $3.06(1.03)$ & - & .021 \\
Positive 2* & $2.92(1.01)$ & $3.31(1.02)$ & - & .044 \\
\hline
\end{tabular}

The values in each cell indicate MEAN (SD). The participants reported higher valence ratings with positive or negative typeface than with the neutral typeface. The values with * mark indicate a statistically significant difference at the .05 level

and $\mathrm{HO}_{2}$ and conclude that the use of typeface in a message can affect and intensify the valence received by users.

\section{Qualitative study: Exploring user experiences}

For qualitative analysis, we used a focus group study. The study was separated into two parts. First, we designed a role-playing study that pairs of participants interacted with each other in prescribed situations. By doing that, we can not only make all the participants get familiar with the system but also see the emotional effect of the typeface in the semistructured situation. After that, we equipped a focus group discussion session where two participants who had interacted with each other and an instructor participated as a moderator. By illustrating the actual user experience, we aimed to find the potential value of the proposed system and leverage the insights from the role-playing study by presenting actual user comments in group discussions.

\subsection{Method}

\subsubsection{Participants}

According to [20], as few as three to six focus groups are likely to identify $90 \%$ of the themes to be observed, we recruited five groups of participants. We recruited five pairs (ten participants; six females, four male) of participants who were friends, and made each pair into one group. They were between the ages of 24 and 34 and were of various nationalities, including American, Chinese, German, Indian, Korean, and Taiwanese. They had sufficient experience using a mobile messenger. English was used as the common language for texting. The session length of the user test was approximately two hours. All participants were compensated with a book voucher worth about US\$20. No users had been exposed to the Emotype application before the experiment. To familiarize users with the system, we provided a short tutorial and then gave them time to use the prototype freely.

\subsubsection{Environment}

The application was run on an Android 4.4 device (7.02-inch screen, $1920 \times 1200$ pixels at 323 ppi). We conducted the user test in various contexts, e.g., lecture room, coffee house, and public lounge. For the role-playing study, a pair of users sat in separate rooms where they could not see or hear each other. For the focus group study, two participants who had 
Table 4 Vignettes for the role-playing test
1 ) Magazine Subscription
(a) A: I like this magazine.
(b) B: So do I. It gives you all the news.
(c) A: Listen, I gave a subscription to my parents.
(d) B:

2) Olympic Season

(a) A: I've been looking forward to this Olympic season so much.

(b) B: Me too! Excitement each day!

(c) A: Anyway, did you watch the soccer game?

(d) B:

3) Winning the Lotto

(a) A: I won the lotto.

(b) B: How much did you win?

(c) A: 5 dollars!

(d) B:
The line (c) has variations in the typeface used

interacted with each other formed a group for the study, and an instructor participated as a moderator.

\subsection{Role-playing study}

\subsubsection{Task}

We designed three vignettes for the role-playing experiment referring to [18] containing the various dialogue examples (see Table 4). The participants in a pair were randomly assigned a role $A$ or $B$. Figure 7 shows an example conversation task. All three vignettes were performed three times, and at each attempt, $A$ was requested to have different feelings and changed the typeface for message (c) (neutral, positive, and negative). Then $B$ replied $(d)$. In the experiment, $B$ surmised about how $A$ felt on message $(c)$, and was requested to submit the option that gave the closest match between negative, positive, or neutral.

\subsubsection{Results}

How the emotion that the recipient inferred changed We analyzed $B$ 's report of the role-playing test. Because five pairs conducted three situations while choosing among three typefaces $(5 \times 3 \times 3)$, we investigated 45 user reports and responses.

Fig. 7 Example conversation task (3. Winning the Lotto)

\begin{tabular}{|c|c|c|c|}
\hline (a) & \multicolumn{3}{|c|}{ A: I won the lotto. } \\
\hline (b) & \multicolumn{3}{|c|}{ B: How much did you win? } \\
\hline \multirow[t]{3}{*}{ (c) } & A & 5 dollars! & Neutral \\
\hline & & 5 dollars! & Positive \\
\hline & & M DULLUARE & Negative \\
\hline (d) & \multicolumn{3}{|c|}{ B: } \\
\hline
\end{tabular}




\begin{tabular}{|c|c|c|c|}
\hline (A) $\begin{array}{r}\text { Reported } \\
\text { feelings } \\
\text { Uspeface }\end{array}$ & neutral & positive & negative \\
\hline neutral & 0.47 & 0.53 & 0.00 \\
\hline positive & 0.13 & 0.87 & 0.00 \\
\hline WEGITOLUE & 0.00 & 0.06 & 0.94 \\
\hline
\end{tabular}

Fig. 8 How well the recipients B guessed the sender A's emotion depending on the typeface used. The sum of each row is one ( $p<.001$ by Fisher's exact test). As we can see the highlighted results (red-dotted boxes), users reported positive feelings with both the neutral typeface and positive typeface with high probability

Figure 8 shows how well the recipients $B$ guessed the sender $A$ 's emotion depending on the typeface used. We observed that, if a sender used the neutral typeface, the recipients guessed the sender's feeling as being neutral or positive almost half the time. However, if a sender changed the typeface, the reported feelings varied significantly depending on the typeface used. Fisher's exact test shows that the result is significant $(p<.001)$.

How the user's reaction changed We analyzed responses that reported the same emotion regardless of the typeface used. From Fig. 8, it is evident that users reported positive feelings with both the neutral typeface and positive typeface (red-dotted boxes). As far as the user reporting is concerned, it seems that the influence of the positive typeface was not much different from that of the neutral typeface. To investigate the effect of the positive typeface, we examined how $B$ 's reaction changed in accordance with typeface usage.

Row (d) in Fig. 9 shows actual response examples by participants who guessed that the sender's intent was positive regardless of which typeface was used (positive or neutral). By reviewing the actual responses, we observed that the positive typeface elicited the more positive response than that of neutral typeface. We can see that users who take role $B$ tended to use emphasizing expressions much more ("so" (P2), "very" (P4), "such a" (P6)) as responses to a message with the positive typeface than to those using the neutral typeface. Even more, the positive words appeared more frequently in the emotional typeface task ("useful" (P4), "good", "happy" (P6), "cool" (P8)). Here, we can associate this to the result from $\mathrm{HO}_{2}$ in our preliminary study. The typeface intensified the valence which a message conveys, then the recipients who perceived the intensified emotion tried to express their emotion more actively as responses to the perceived emotion.

\subsection{Focus group discussions}

In the focus group discussion, participants discussed their diverse experiences with regard to given special message examples. In addition to that, we explore values of Emotype via conversations obtained from free chats between participants.

\subsubsection{Emotion words, emoji, and typefaces in texting}

We provided message examples to participants which were equivalent in contents, but different in the way expressing emotion. Figure 10 shows an example. Then they reported the differences between emotion words, emoji, and typefaces. 


\begin{tabular}{|c|c|c|c|c|}
\hline (a) & A: & \multicolumn{2}{|l|}{ I like this magazine } & $\rightarrow(\mathrm{P} 1 / 3 / 5 / 7)$ \\
\hline (b) & B: & \multicolumn{2}{|c|}{ So do I. It gives you all the news. } & $\rightarrow(\mathrm{P} 2 / 4 / 6 / 8)$ \\
\hline (c) & A: & $\begin{array}{l}\text { "Listen, I gave a } \\
\text { subscription of a maga- } \\
\text { zine to my parents." }\end{array}$ & $\begin{array}{l}\text { "listen. i gave a subscrip- } \\
\text { tion of a magazine to my } \\
\text { parents." }\end{array}$ & $\rightarrow(\mathrm{P} 1 / 3 / 5 / 7)$ \\
\hline \multirow{4}{*}{ (d) } & \multirow{4}{*}{ B: } & Nice, how do they like it? & So nice ;) they must like it & $\rightarrow(\mathrm{P} 2)$ \\
\hline & & $\begin{array}{l}\text { Oh, that's great. } \\
\text { Did they like it? }\end{array}$ & $\begin{array}{l}\text { Nice! :) did they like it? I guess } \\
\text { it is very useful for them }\end{array}$ & $\rightarrow(\mathrm{P} 4)$ \\
\hline & & Subscription? & $\begin{array}{l}\text { You are such a good girl! Th } \\
\text { ey must be happy about it! }\end{array}$ & $\rightarrow(\mathrm{P} 6)$ \\
\hline & & Why? & That's cool & $\rightarrow(\mathrm{P} 8)$ \\
\hline
\end{tabular}

Fig. 9 The actual user responses in the vignettes 1. Magazine Subscription. We observe that the use of the positive typeface elicited an active response in row (d). We highlighted emphasizing expressions in bold, and colored the positive words green. For example, in row P1, with the neutral typeface, P1 simply gave a positive response (Nice,), and asked (how do they like it?). However, with the positive typeface, P1 showed a more active positive response (So nice;)) and affirming (they must like it). Here, $P_{i}$ indicates the id of each participant $(i=1,2,3, \ldots, 8)$

Findings All participants reported that the positive emotion conveyed by neutral fonts (Fig. 10(3)) evoked formal and business-like feelings. On the other hand, they felt that the speaker genuinely seemed happy in the messages that used emoji and typefaces. "Both the emoji and typeface conveyed very positive feelings, and the message with the typeface sounded more intimate" (P4). The comparison between typeface, emoji, and emotion word usages revealed that both typeface and emoji conveyed emotion effectively, but the nuance between them was slightly different. "With the emoji, I felt the sender was smiling after she or he says the words, but with the typeface, I felt the sender was saying it in a happy tone of voice all along" (P6).

We surmise the observations comes from inner voice experience [23] — speech rehearsal in one's mind. In other words, when people read the message with typeface, the unique visual appearance affects their inner voice experience and then would give them feelings such as a happy tone of voice. On the other hand, in case of negative messages, nonverbal signals in emoji and typeface eased the negative mood in the chat. All participants mentioned that negative feelings conveyed by text only evoked more negative feelings than the emoji and typeface. "If I were really in negative mood, I would not use emoji or typefaces. But if I didn't want to make others worry about me, a message with the typeface would seem to be effective" (P5). Some participants also reported that they felt a tone of voice that guides them to speaker's personality with Emotype: "The message with negative typeface sounded like someone's voice who has a violent temper" (P6).

Fig. 10 An example using three different types of emotional expression: Emoji, Emotype and emotion word
(1) Wow, that's great! $=$

(2) wow. that's great!

(3) Wow, that's great! I'm glad to hear that. 


\begin{tabular}{|c|c|c|c|c|}
\hline & Content & Typeface & & Inconsistency \\
\hline (1) & Positive & Positive & i am happy these days! & \\
\hline (2) & Positive & Negative & 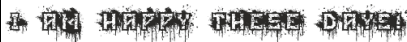 & $\checkmark$ \\
\hline (3) & Negative & Negative & 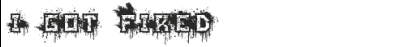 & \\
\hline (4) & Negative & Positive & i got fired & $\checkmark$ \\
\hline
\end{tabular}

Fig. 11 Messages showing inconsistency between the content and the typeface

\subsubsection{Inconsistency between the content and typeface}

It is known that emotional conflict tasks draw unusual behaviors because of the implicit emotion regulation [21]. We therefore investigated how inconsistencies between the content and the typeface affected the participants' feelings and thoughts. All participants received four short messages (Fig. 11) and were asked to report how they felt in response to the messages. The content and typeface of message (1) were both positive; however, message (2) had positive content and negative typeface, therefore showing inconsistency. In the same way, the message (4) also shows inconsistency.

Findings One of the functions of messages that showed inconsistency between the content and typeface was humor in a sarcastic situation. According to participants P1 and P2: "It reminded of me the TV animation, South Park, in that expression of a sense of humor." In addition to P1 and P2, all other participants mentioned that they experienced a humorous feeling from the sarcastic situation. It seemed that the inconsistency in a single media only caused confusion; however, the inconsistency between the two media, e.g., a positive text but negative typeface, was perceived as a humorous mood by the recipient. Some participants described it as follows: "If I received the message [I am happy and angry], it only makes me confused. But with the message with (2) [I am happy (with anger font)], it seems funny. It seems like the sender is trying to make the mood humorous even though he or she was really upset" (P5, P6). The use of typeface also enabled participants to imagine the situation in detail: "From the message, [I got fired] with a happiness typeface, I imagined a situation in which the sender was fired from a job she or he really disliked" (P7, P8).

These reports implied that the inconsistency between the text and typeface was perceived by users as humorous feeling and the use of typeface contributes to rich emotional experiences.

\subsubsection{Dynamic tone change in free chat}

It is known that seriatim transmissions of parts of a message are characteristic of instant messages (e.g., hey man [send] what's up [send]) [6]. In the free chats between participants, we discovered that this characteristic brought about interesting Emotype usage.

Findings Figure 12 shows an interesting example of usage. Participants preferred to express different emotional signals in accordance with the segment of the message. They actively utilized typeface changes in seriatim messages. This usage fulfilled the need to disclose the sender's feeling or intention. P1 explained why he showed a dynamic tone change between the messages P1(1) and P1(2), "I used the positive typeface to warn about the negative 


\section{P2(1): I want to quit studies and open an Indian restaurant \\ P2(2): how do u think about the idea? \\ P2(3): it's my new goal

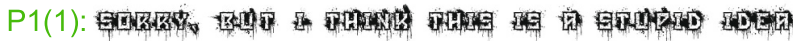 \\ P1(2): one day it will pay off}

Fig. 12 An free chat example which shows dynamic tone changes

future that P2 may have, but not to make the mood too serious" (P1). This kind of usage also observed in other participants' conversations.

As we observed in the free chats, the dynamic usage of typefaces in seriatim transmissions of messages enabled the expression of multiple emotions in a message and conferred a lively mood in the conversation. Furthermore, typefaces were being used naturally to create a relaxed atmosphere to the chat.

\section{Discussion and conclusions}

This study has proposed an approach that employs typefaces to convey intended emotional states in a mobile environment. To examine the effectiveness of this approach, we designed user studies. In our preliminary study, we demonstrated the feasibility of using typefaces to communicate emotions. If the target message was ambivalent, the perceived emotion varied greatly depending on the typefaces used. This result indicates that emotions are being transmitted through another channel- the typeface, even if the emotions are not clearly stated in the text. If the emotion of a message was explicitly mentioned, the use of a typeface that matched the emotion of the message emphasized the emotion. In the user study, we explored the user experiences with role-playing and focus group discussion studies. We observed that the use of typeface not only modulates the emotional signals a message conveyed, but also elicits active responses in expressing emotions. We also obtained various user experience reports. We observed that there was a slight difference in nuance between typeface and emoticon, and participants reported this as acoustic experiences. The inconsistency between the content and typeface created a humorous feeling and enabled participants to imagine the situation in detail. In the free chats between participants, we observed that they naturally exploited typeface to guide a conversation toward an intended mood. Here, typeface contributed to create rich experiences such as dynamic tone changes. The series of comments from the focus group discussions suggested that the Emotype conveys nonverbal signals, such as a tone of voice. In other words, Emotype was demonstrating "how it was said" in the chats. Our findings indicate that the use of typefaces in mobile communication will expand channels of nonverbal signals and contribute to users' mobile communication.

However, several limitations remain. We mainly investigated the emotional effect of two typefaces, negative and positive. The emotional effects of other typefaces need to be examined. Another issue comes from individual differences. Even if there was a strong consensus on the emotional effect of a given typeface, these effects could vary according to the user. Finally, we could not observe user behaviors in natural situations. Because the participants knew that conversations will be analyzed, it was difficult for them to chat naturally. This eventually led our user study to be conducted in the limited situation. We hope that Emotype 
will be applied to real messenger applications, and expect to find further values through a long-term study like emoji/emoticon researches have done.

\section{Acknowledgements This research is partially supported by KAKENHI 17K19963.}

Open Access This article is distributed under the terms of the Creative Commons Attribution 4.0 International License (http://creativecommons.org/licenses/by/4.0/), which permits unrestricted use, distribution, and reproduction in any medium, provided you give appropriate credit to the original author(s) and the source, provide a link to the Creative Commons license, and indicate if changes were made.

Publisher's Note Springer Nature remains neutral with regard to jurisdictional claims in published maps and institutional affiliations.

\section{References}

1. Ali AZM, Wahid R, Samsudin K, Idris MZ (2013) Reading on the computer screen: does font type has effects on web text readability? International Education Studies 6(3):26

2. Alm CO, Roth D, Sproat R (2005) Emotions from text: machine learning for text-based emotion prediction. In: Proceedings of the conference on human language technology and empirical methods in natural language processing, Association for Computational Linguistics, pp 579-586

3. Amare N, Manning A (2012) Seeing typeface personality: emotional responses to form as tone. In: 2012 IEEE International professional communication conference, IEEE, pp 1-9

4. Arditi A, Cho J (2005) Serifs and font legibility. Vis Res 45(23):2926-2933

5. Azadi S, Fisher M, Kim V, Wang Z, Shechtman E, Darrell T (2018) Multi-content gan for few-shot font style transfer. In: Proceedings of the IEEE conference on computer vision and pattern recognition, vol 11. $\mathrm{p} 13$

6. Baron NS (2004) See you online gender issues in college student use of instant messaging. J Lang Soc Psychol 23(4):397-423

7. Byron K, Baldridge DC (2007) E-mail recipients' impressions of senders' likability the interactive effect of nonverbal cues and recipients' personality. J Bus Commun 44(2):137-160

8. Candello H, Pinhanez C, Figueiredo F (2017) Typefaces and the perception of humanness in natural language chatbots. In: Proceedings of the $2017 \mathrm{CHI}$ conference on human factors in computing systems, ACM, pp 3476-3487

9. Chen X, Bennett PN, Collins-Thompson K, Horvitz E (2013) Pairwise ranking aggregation in a crowdsourced setting. In: Proceedings of the sixth ACM international conference on Web search and data mining, ACM, pp 193-202

10. Childers TL, Jass J (2002) All dressed up with something to say: effects of typeface semantic associations on brand perceptions and consumer memory. J Consum Psychol 12(2):93-106

11. Choi S, Aizawa K, Sebe N (2018) Fontmatcher: font image paring for harmonious digital graphic design. In: 23rd international conference on intelligent user interfaces, ACM, pp 37-41

12. Choi S, Yamasaki T, Aizawa K (2016) Typeface emotion analysis for communication on mobile messengers. In: Proceedings of the 1st international workshop on multimedia alternate realities, ACM, pp 37-40

13. Co. L (2016) Line stickers. LINE STORE. https://store.line.me/stickershop/showcase/top//en

14. Collins NL, Miller LC (1994) Self-disclosure and liking: a meta-analytic review. Psychol Bull 116(3):457

15. Derks D, Fischer AH, Bos AE (2008) The role of emotion in computer-mediated communication: a review. Comput Hum Behav 24(3):766-785

16. de Freitas LA, Vanin AA, Hogetop DN, Bochernitsan MN, Vieira R (2014) Pathways for irony detection in tweets. In: Proceedings of the 29th annual ACM symposium on applied computing, ACM, pp 628-633

17. Ekman P (1992) An argument for basic emotions. Cogn Emot 6(3-4):169-200

18. Esl fast. http://www.eslfast.com/easydialogs/ (2017). Accessed: 2017-06-28

19. Gottschalk LA, Gleser GC (1969) The measurement of psychological states through the content analysis of verbal behavior. University of California Press, Berkeley

20. Guest G, Namey E, McKenna K (2017) How many focus groups are enough? building an evidence base for nonprobability sample sizes. Field methods 29(1):3-22 
21. Gyurak A, Gross JJ, Etkin A (2011) Explicit and implicit emotion regulation: a dual-process framework. Cogn Emot 25(3):400-412

22. Hancock JT, Landrigan C, Silver C (2007) Expressing emotion in text-based communication. In: Proceedings of the SIGCHI conference on Human factors in computing systems, ACM, pp 929932

23. Heery MW (1989) Inner voice experiences: an exploratory study of thirty cases. J Transpers Psychol 21(1):73

24. Henderson PW, Giese JL, Cote JA (2004) Impression management using typeface design. J Mark 68(4):60-72

25. Huang S, Zhong Z, Jin L, Zhang S, Wang H (2018) Dropregion training of inception font network for high-performance chinese font recognition. Pattern Recogn 77:395-411

26. Inc. G (2016) Allo. Apps. https://play.google.com/store/apps/details?id=com.google.android.apps. fireball\&hl=en

27. Kalman YM, Gergle D (2014) Letter repetitions in computer-mediated communication: a unique link between spoken and online language. Comput Hum Behav 34:187-193

28. Kim M, Choi K, Suk HJ (2016) Yo!: Enriching emotional quality of single-button messengers through kinetic typography. In: Proceedings of the 2016 ACM conference on designing interactive systems, DIS '16. ACM, New York, pp 276-280. https://doi.org/10.1145/2901790.2901835

29. Kim YE, Schmidt EM, Migneco R, Morton BG, Richardson P, Scott J, Speck JA, Turnbull D (2010) Music emotion recognition: a state of the art review. In: Proceedings of the ISMIR, Citeseer, pp 255-266

30. Layer (2016) Atlas. https://atlas.layer.com/

31. Lee J, Jun S, Forlizzi J, Hudson SE (2006) Using kinetic typography to convey emotion in text-based interpersonal communication. In: Proceedings of the 6th conference on designing interactive systems, DIS '06. ACM, New York, pp 41-49. https://doi.org/10.1145/1142405.1142414

32. Li Y, Suen CY (2010) Typeface personality traits and their design characteristics. In: proceedings of the 9th IAPR international workshop on document analysis systems, ACM, pp 231-238

33. Limited CG (2016) Font infinity. iTunes. https://itunes.apple.com/us/app/font-infinity-cool-new-fonts/ id $885791898 ? \mathrm{mt}=8$

34. Machajdik J, Hanbury A (2010) Affective image classification using features inspired by psychology and art theory. In: Proceedings of the 18th ACM international conference on Multimedia, ACM, pp 83-92

35. Mehrabian A (1980) Basic dimensions for a general psychological theory implications for personality, social, environmental and developmental studies

36. Min F (2016) Font dresser. iTunes. https://itunes.apple.com/us/app/font-dresser-for-ext-editing/ id $467286515 ? \mathrm{mt}=8$

37. Montefinese M, Ambrosini E, Fairfield B, Mammarella N (2014) The adaptation of the affective norms for english words (anew) for italian. Behav Res Methods 46(3):887-903

38. O'Donovan P, Líbeks J, Agarwala A, Hertzmann A (2014) Exploratory font selection using crowdsourced attributes. ACM Trans Graph (TOG) 33(4):92

39. Oyama T (2003) Affective and symbolic meanings of color and form: experimental psychological approaches. Empir Stud Arts 21(2):137-142

40. Park TW, Kim SJ, Lee G (2014) A study of emoticon use in instant messaging from smartphone. In: International conference on human-computer interaction, Springer, pp 155-165

41. Rello L, Pielot M, Marcos MC (2016) Make it big!: the effect of font size and line spacing on online readability. In: Proceedings of the $2016 \mathrm{CHI}$ conference on human factors in computing systems, CHI '16. ACM, New York, pp 3637-3648. https://doi.org/10.1145/2858036.2858204

42. Rubinstein R (1988) Digital typography: an introduction to type and composition for computer system design. Addison-wesley Longman Publishing Co. Inc

43. Shaikh AD, Chaparro BS, Fox D (2006) Perception of fonts: perceived personality traits and uses. Usability News 8(1):1-6

44. Tewell J, Bird J, Buchanan GR (2017) The heat is on: a temperature display for conveying affective feedback. In: Proceedings of the 2017 CHI conference on human factors in computing systems, ACM, pp 1756-1767

45. Walther JB, Loh T, Granka L (2005) Let me count the ways the interchange of verbal and nonverbal cues in computer-mediated and face-to-face affinity. J Lang Soc Psychol 24(1):36-65

46. Wang Z, Yang J, Jin H, Shechtman E, Agarwala A, Brandt J, Huang TS (2015) Deepfont: identify your font from an image. In: Proceedings of the 23rd ACM international conference on Multimedia, ACM, pp $451-459$

47. Zhang Y, Zhang Y, Cai W (2018) Separating style and content for generalized style transfer. In: Proceedings of the IEEE conference on computer vision and pattern recognition, vol 1 


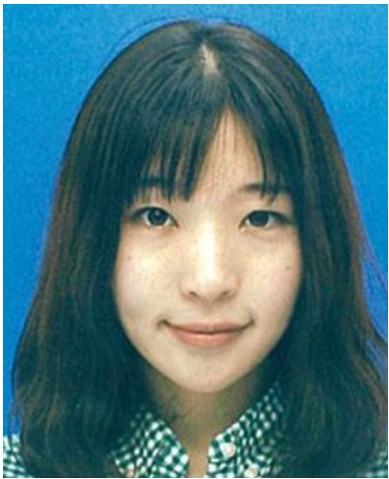

Saemi Choi received the B.E degree in Electrical Engineering from Inha University, Incheon, South Korea, in 2012 and the M.S.A. degree in Interdisciplinary Information Studies from University of Tokyo, Tokyo, Japan, in 2015. She is currently pursuing the Ph.D. degree in Interdisciplinary Information Studies from the University of Tokyo. Her research interests include multimedia, image processing, affective computing and human computer interaction.

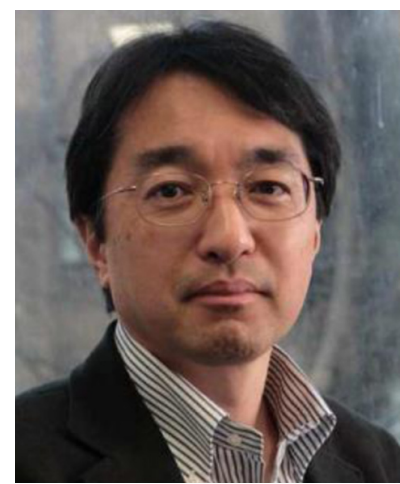

Prof. Kiyoharu Aizawa received the B.E., the M.E., and the Dr.Eng. degrees in Electrical Engineering all from the University of Tokyo, in 1983, 1985, 1988, respectively. He is currently a Professor at Department of Information and Communication Engineering of the University of Tokyo. He was a Visiting Assistant Professor at University of Illinois from 1990 to 1992. His research interest is in image processing and multimedia applications. He received the 1987 Young Engineer Award and the 1990, 1998 Best Paper Awards, the 1991 Achievement Award, 1999 Electronics Society Award from IEICE Japan, and the 1998 Fujio Frontier Award, the 2002 and 2009 Best Paper Award, and 2013 Achievement award from ITE Japan. He received the IBM Japan Science Prize in 2002. He is currently a Senior Associate Editor of IEEE Tras. Image Processing, and on Editorial Board of ACM TOMM, APSIPA Transactions on Signal and Information Processing, and International Journal of Multimedia Information Retrieval. He served as the Editor in Chief of Journal of ITE Japan, an Associate Editor of IEEE Trans. Image Processing, IEEE Trans. CSVT and IEEE Trans. Multimedia. He has served a number of international and domestic conferences; he was a General co-Chair of ACM Multimedia 2012. He is a council member of Science Council of Japan. 